\title{
Sex differences in the immune response to acute COVID-19 respiratory tract infection
}

\author{
Shaohua Qi ${ }^{1 \dagger}$, Conelius Ngwa ${ }^{1 \dagger}$, Diego A. Morales Scheihing ${ }^{1}$, Abdullah Al Mamun ${ }^{1}$, Hilda W. Ahnstedt ${ }^{1}$, \\ Carson E. Finger', Gabriela Delevati Colpo', Romana Sharmeen', Youngran Kim', HuiMahn A. Choi², \\ Louise D. McCullough ${ }^{1}$ and Fudong Liu ${ }^{1 *}$ (])
}

\begin{abstract}
Background: Sex differences in COVID-19 are increasingly recognized globally. Although infection rates are similar between the sexes, men have more severe illness. The mechanism underlying these sex differences is unknown, but a differential immune response to COVID-19 has been implicated in several recent studies. However, how sex differences shape the immune response to COVID-19 remains understudied.
\end{abstract}

Methods: We collected demographics and blood samples from over 600 hospitalized patients diagnosed with COVID-19 from May 24th 2020 to April 28th, 2021. These patients were divided into two cohorts: Cohort 1 was further classified into three groups based on the severity of the disease (mild, moderate and severe); Cohort 2 patients were longitudinally followed at three time points from hospital admission (1 day, 7 days, and 14 days). MultiPlex and conventional ELISA were used to examine inflammatory mediator levels in the plasma in both cohorts. Flow cytometry was conducted to examine leukocyte responses in Cohort 2.

Results: There were more COVID ${ }^{+}$males in the total cohort, and the mortality rate was higher in males vs. females. More male patients were seen in most age groups (in 10-year increments), and in most ethnic groups. Males with severe disease had significantly higher levels of pro-inflammatory cytokines (IL-6, IL-8, MCP-1) than females; levels of IL-8, GRO, sCD40L, MIP-1 $\beta$, MCP-1 were also significantly higher in severe vs. mild or control patients in males but not in females. Females had significantly higher anti-inflammatory cytokine IL-10 levels at 14 days compared to males, and the level of IL-10 significantly increased in moderate vs. the control group in females but not in males. At 7 days and 14 days, males had significantly more circulating neutrophils and monocytes than females; however, B cell numbers were significantly higher in females vs. males.

Conclusion: Sex differences exist in hospitalized patients with acute COVID-19 respiratory tract infection. Exacerbated inflammatory responses were seen in male vs. female patients, even when matched for disease severity. Males appear to have a more robust innate immune response, and females mount a stronger adaptive immune response to COVID-19 respiratory tract infection.

Keywords: COVID-19, Cytokine, Leukocytes, Immune response, Sex difference

*Correspondence: Fudong.Liu@uth.tmc.edu

†'Shaohua Qi and Conelius Ngwa have contributed equally to this work ${ }^{1}$ Department of Neurology, McGovern Medical School, The University of Texas Health Science Center at Houston, 6431 Fannin Street, Houston, TX 77030, USA

Full list of author information is available at the end of the article

\section{Background}

Coronavirus Disease 2019 (COVID-19) caused by the severe acute respiratory syndrome coronavirus 2 (SARSCoV-2) has been a worldwide pandemic, with over 32 million people in the US infected as of May 2021 [1]. About $20 \%$ of those infected with COVID-19 require original author(s) and the source, provide a link to the Creative Commons licence, and indicate if changes were made. The images or other third party material in this article are included in the article's Creative Commons licence, unless indicated otherwise in a credit line to the material. If material is not included in the article's Creative Commons licence and your intended use is not permitted by statutory regulation or exceeds the permitted use, you will need to obtain permission directly from the copyright holder. To view a copy of this licence, visit http://creativecommons.org/licenses/by/4.0/. The Creative Commons Public Domain Dedication waiver (http://creativeco mmons.org/publicdomain/zero/1.0/) applies to the data made available in this article, unless otherwise stated in a credit line to the data. 
hospitalization, of which $5-10 \%$ require intensive care therapy. The COVID-19 virus is transmitted through respiratory droplets from coughing and sneezing, and enters the nasal system to replicate and propagate. There has been a lack of efficacious therapies for the virus, and thus the immune system remains the best defense [2]. Once the human body is infected, immune cells are mobilized, and in many cases "cytokine storm" occurs in an effort to combat the virus [3]. It is increasingly recognized that COVID-19 disproportionately affects men compared to women $[4,5]$. Preliminary studies have suggested that while the prevalence of infection is the same in men and women, male patients are more likely to be hospitalized, have a more severe course of disease and higher mortality [6]. Sex differences are well known in innate and adaptive immunity with resultant sex-specific responses to vaccines and infections [7], which has been postulated as an important reason for the differential response across sex to COVID-19.

To better understand the profiles of sex differences in immune responses to COVID-19, in this study we examined the plasma inflammatory mediators and peripheral blood immune cells from hospitalized patients (both sexes) with COVID-19, and at multiple time points. We quantified immune cell mobilization not only based on the disease severity, but also longitudinally.

\section{Methods}

\section{Sampling and cohorts}

Samples were collected from consented healthy donors and hospitalized patients with acute COVID-19 respiratory tract infection (at the Memorial Hermann Hospital System) from May 24th 2020 to April 28th, 2021 (see Additional file 1 for the Inclusion/Exclusion Criteria). The average age of these patients was $53.49 \pm 16.89$, with the majority being between 41 and 65 years old (age ranged from 19 to 101). Males constituted 60.0\% of the sample ( $N=350$ male and 276 females). American Indians or Alaska Natives constituted $0.16 \%$, Asians 3.14\%, African Americans 21.9\%, Hispanics 4.4\%, Whites $62.9 \%$, and unable to determine (UTD) $7.5 \%$ of patients. All collected samples were divided into two cohorts as a smaller subset of patients were hospitalized for over 2 weeks. Cohort 1 patients were graded for severity based on respiratory symptoms and the need for supplemental oxygen therapy according to the classification from the NIH COVID-19 spectrum [8] and the therapeutic management of COVID-19 [9]: mild, nasal cannula less than $5 \mathrm{~L}$ and less than 5 days in the hospital; moderate, nasal cannula more than $5 \mathrm{~L}$ or high-flow oxygen therapy and more than 5 days in the hospital; severe, ventilator or died because of COVID-19. Blood samples from Cohort 1 patients were collected within 3-4 days of hospital admission, and Cohort 2 samples were collected on day 1, day 7, and day 14 from admission. Briefly, the blood sample $(5 \mathrm{~mL})$ was obtained by venipuncture or from an existing line cleared of I.V. solution (venipuncture, arterial or venous recorded) in 6-mL K2 EDTA Vacutainer tubes (BD, Franklin Lakes, NJ, USA). Samples were processed within one hour of collection. Blood cells were removed by centrifugation $\left(4{ }^{\circ} \mathrm{C}, 800 \times g\right.$ for $\left.10 \mathrm{~min}\right)$, and the platelet-poor plasma collected after another centrifugation $\left(4{ }^{\circ} \mathrm{C}, 10,000 \times g\right.$ for $\left.10 \mathrm{~min}\right)$ and stored at -80 ${ }^{\circ} \mathrm{C}$ until all samples were ready for analysis. All assessments were performed blinded to the subject's history of COVID-19. The study was approved by the Institutional Review Board (IRB) of University of Texas Health Science Center at Houston (HSC-MS-17-0452).

\section{Multiplex and conventional ELISA}

Multiplex enzyme-linked immunosorbent assays were performed on plasma of Cohort 1 for inflammatory mediators including cytokines and chemokines using a commercially available panel (MILLIPLEX ${ }^{\circledR}$ Human Cytokine/Chemokine/Growth Factor Panel A-Immunology Multiplex Assay HCYTA-60K-08, Millipore Sigma) by following the manufacturer's instructions. The inflammatory mediators in the panel of the kit are: sCD40L, EGF, Eotaxin, FGF-2, Flt-3 ligand, Fractalkine, G-CSF, GM-CSF, GRO $\alpha$, IFN $\alpha 2$, IFN $\gamma$, IL- $1 \alpha$, IL- $1 \beta$, IL1ra, IL-2, IL-3, IL-4, IL-5, IL-6, IL-7, IL-8, IL-9, IL-10, IL-12 (p40), IL-12 (p70), IL-13, IL-15, IL-17A, IL-17E/ IL-25, IL-17F, IL-18, IL-22, IL-27, IP-10, MCP-1, MCP-3, M-CSF, MDC (CCL22), MIG, MIP-1 $\alpha$, MIP-1 $\beta$, PDGFAA, PDGF-AB/BB, RANTES, TGF $\alpha$, TNF $\alpha$, TNF $\beta$, and VEGF-A (see Additional file 2 for details).

Conventional ELISA was used to examine IL-6, IL-8, IL-10, TNF- $\alpha$, and TGF- $\beta 1$ in plasma of Cohort 2 patients at three time points ( 1 day, 7 days, 14 days) by using human ELISA LEGEND MAX (IL-6_430507, IL-8_431507, IL-10_430607, TNF- $\alpha \_430207$, and TGF$\beta 1$ 436707; BioLegend). Signals were read at $450 \mathrm{~nm}$ in EnSpire ${ }^{\text {TM }}$ Multimode Plate Reader (PerkinElmer, Inc.).

\section{Flow cytometry}

Flow cytometry assay was conducted on the blood samples of Cohort 2. Briefly, each human blood aliquot $(100 \mu \mathrm{L})$ in autoclaved Eppendorf tube was added pre-chilled HBSS containing $2 \%$ FBS $(1 \mathrm{~mL})$, and then followed by centrifuging at $500 \times g$ for $5 \mathrm{~min}$ and at 4 ${ }^{\circ} \mathrm{C}$. The pellets (cells) were re-suspended by adding another pre-chilled HBSS containing $2 \%$ FBS $(200 \mu \mathrm{L})$ and blocked in human Fc Block (422302, Biosciences) ( $1 \mu \mathrm{L} /$ sample). For live/dead cell discrimination, a fixable viability dye lock, carboxylic acid succinimidyl ester was used (CASE-AF350, Invitrogen). Next, the cells 
were stained by adding the antibody mix specific for each cell population (Tables 1, 2 and 3) and incubated for $30 \mathrm{~min}$ at $4{ }^{\circ} \mathrm{C}$ on a rotator in the dark and at $4{ }^{\circ} \mathrm{C}$. Isotype control antibodies were used in parallel with the cell specific antibodies to remove the nonspecific signals in each panel. After antibody incubation, the samples were suspended in pre-chilled HBSS containing $2 \% \mathrm{FBS}(1 \mathrm{~mL})$ and centrifuged at $500 \times \mathrm{g}$ for $5 \mathrm{~min}$ and at $4{ }^{\circ} \mathrm{C}$. The pellets (cells) were suspended again and fixed in ice-cold 1\% PFA $(200 \mu \mathrm{L})$ for $10 \mathrm{~min}$ and in the dark. Following another wash, red blood cells were lysed by adding $2 \mathrm{~mL}$ of $1 \times \mathrm{RBC}$ Lysis buffer (420302, BioLegend) at room temperature (Thermo Fisher Scientific, Waltham, MA, USA) for $10 \mathrm{~min}$. The samples were washed again and centrifuged at $500 \times g$ for 5 min and at $4{ }^{\circ} \mathrm{C}$, and then re-suspended in pre-chilled HBSS containing $2 \%$ FBS $(300 \mu \mathrm{L})$, and immediately assayed in a flow cytometer (CytoFLEX b75408, Beckman Coulter). Data were analyzed by using FlowJo_v10.7.2 (Tree Star Inc.).

\section{Statistical analyses}

Cytokine and immune cell data were presented as the mean \pm standard error of the mean (SEM), and comparisons were made with the severity of COVID-19 and sex using two-way ANOVA plus Sidak's post hoc correction. Prevalence of other organ involvement or comorbidities, in addition to pneumonia, were presented as $\%$ of patients with $95 \%$ confidence intervals according to the severity of COVID-19, time point since admission, and sex. Statistical differences between the sexes were determined using Chi-square tests and any trends across the levels of severity or follow-up time points were assessed using the extended Mantel-Haenszel Chi-square for linear trend. All statistical data analysis was performed using Graph-Pad PRISM Version 9.0 (GraphPad Software, CA, USA) and statistically significance levels were set at $p<0.05$ for 2 -tailed tests.

Table 1 Antibody panel for neutrophil flow

\begin{tabular}{lll}
\hline Antibody & Source & Catalog no. \\
\hline $\begin{array}{l}\text { BUV395 mouse anti-human CD45 } \\
\text { antibody }\end{array}$ & BD Biosciences & 563792 \\
$\begin{array}{l}\text { BV421 mouse anti-human CD11b } \\
\text { antibody }\end{array}$ & BD Biosciences & 562632 \\
$\begin{array}{l}\text { 6g8-AF568 anti-DEspR antibody } \\
\text { LIVE/DEAD }\end{array}$ & LakePharma & Nixable aqua dead cell stain \\
kit & Thermo Fisher & L-34966 \\
\hline
\end{tabular}

Table 2 Antibody panel for lymphocyte flow

\begin{tabular}{|c|c|c|}
\hline Antibody & Source & Catalog no. \\
\hline $\begin{array}{l}\text { BUV395 mouse anti-human CD45 } \\
\text { antibody }\end{array}$ & BD Biosciences & 563792 \\
\hline $\begin{array}{l}\text { BV421 mouse anti-human CD11b } \\
\text { antibody }\end{array}$ & BD Biosciences & 562632 \\
\hline $\begin{array}{l}\text { Brilliant Violet } 421^{\mathrm{TM}} \text { anti-human CD3 } \\
\text { antibody }\end{array}$ & BioLegend & 317344 \\
\hline PE anti-human CD19 antibody & BioLegend & 392506 \\
\hline $\begin{array}{l}\text { LIVE/DEAD }{ }^{\mathrm{TM}} \text { fixable aqua dead cell stain } \\
\text { kit }\end{array}$ & Thermo Fisher & L-34966 \\
\hline
\end{tabular}

Table 3 Antibody panel for monocyte flow

\begin{tabular}{|c|c|c|}
\hline Antibody & Source & Catalog no. \\
\hline $\begin{array}{l}\text { BUV395 mouse anti-human CD45 } \\
\text { antibody }\end{array}$ & BD Biosciences & 563792 \\
\hline $\begin{array}{l}\text { Brilliant Violet } 785^{\mathrm{TM}} \text { anti-human CD16 } \\
\text { antibody }\end{array}$ & BioLegend & 302046 \\
\hline $\begin{array}{l}\text { LIVE/DEAD }{ }^{\mathrm{TM}} \text { fixable aqua dead cell stain } \\
\text { kit }\end{array}$ & Thermo Fisher & L-34966 \\
\hline APC anti-human CD14 antibody & BioLegend & 367118 \\
\hline PE anti-human CD24 antibody & BioLegend & 311106 \\
\hline
\end{tabular}

\section{Results}

Male sex is a risk factor for acute COVID-19 respiratory tract infection

A sex imbalance in the hospitalization and mortality from COVID-19 patients has been reported worldwide [10-14]. We examined the sex (self-identified) distribution in our hospitalized patients that were graded into three groups (mild, moderate and severe) [8]. There were more males than females in the mild and severe group (Fig. 1A, B). We also analyzed the male/female ratio by age in 10-year increments. In most age groups, the ratios were above one, indicating more male vs. female patients (Fig. 1C). Interestingly, when analyzed based on ethnicity, the male/female ratios were above one in almost all ethnicities except for African Americans (Fig. 1D). The overall mortality rate was higher in male vs. female patients (Fig. 1E).

\section{Inflammatory mediator levels in plasma after acute COVID-19 respiratory tract infection}

Previous studies suggested that the severity of COVID19 is associated with an increased level of inflammatory mediators including cytokines and chemokines $[15,16]$. Here, we examined 48 mediators (Additional file 2) in Cohort 1 patients, among which 38 were detectable by our Multiplex ELISA assay. We presented the data 

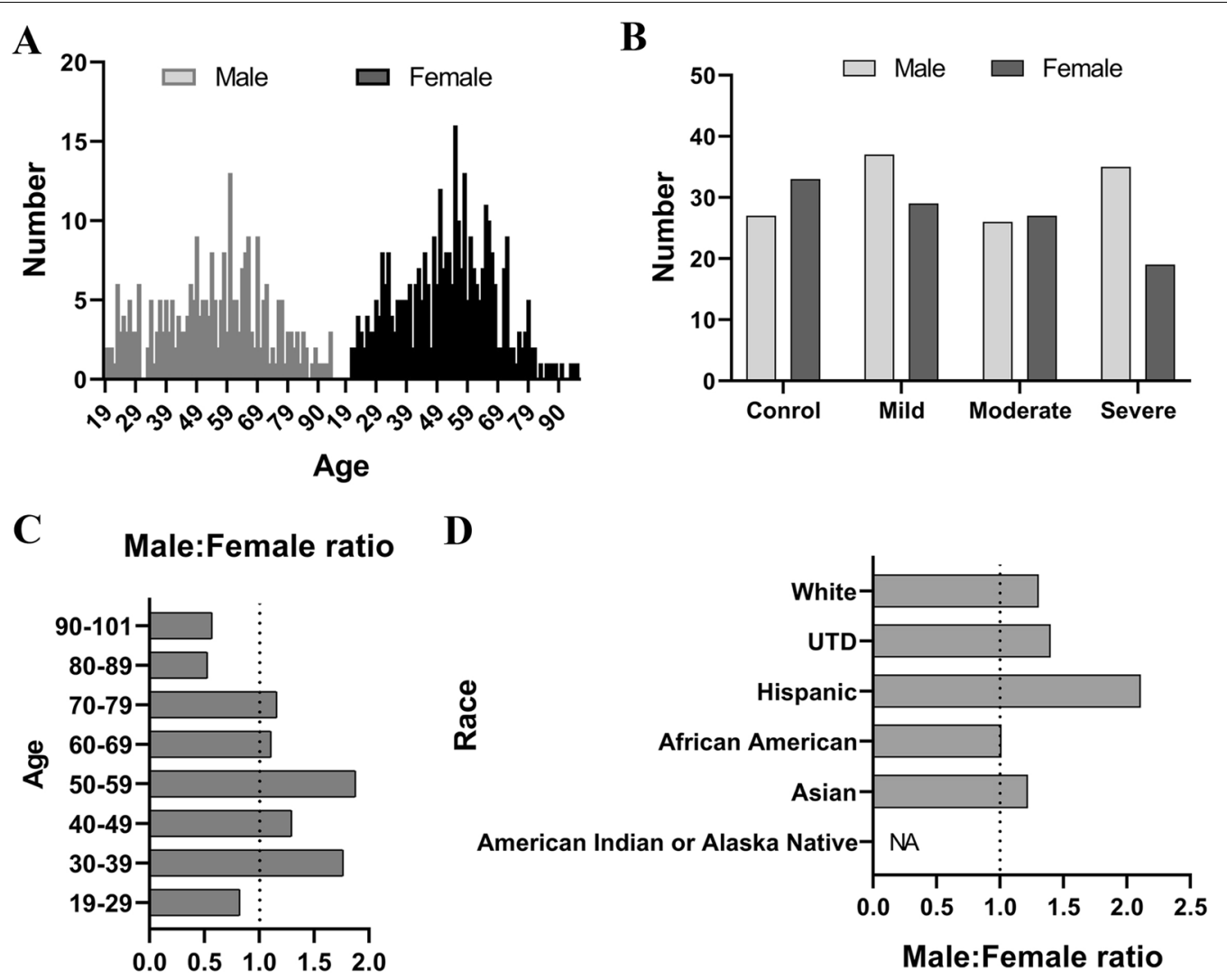

\section{E Mortality}

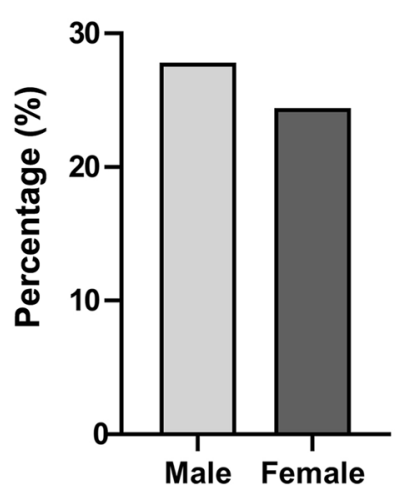

Fig. 1 Male sex is a risk factor for acute COVID-19 respiratory tract infection. A Age distribution of COVID-19 patients. B Male and female patients based on clinical classification of severity of COVID-19. C Male-to-female ratios of COVID-19 cases in 10 years of age increments. D Male-to-female ratios of COVID-19 cases based on ethnicity. E Mortality rate in males vs. females

of TNF- $\alpha$, IL-6, IL-8, Eotaxin, IL-10, MCP-3, MDC (CCL22), GRO $\alpha$, IP-10, sCD40L, MIP-1 $\beta$, and Fractalkine only, as differences between groups were found. However, data of other mediators (EGF, FGF-2, Flt-3 ligand, G-CSF, GM-CSF, IFN $\alpha 2$, IFN $\gamma$, IL- $1 \alpha$, IL- $1 \beta$, IL1ra, IL-2, IL-3, IL-4, IL-5, IL-7, IL-9, IL-12 (p40), IL-12 (p70), IL-13, IL-15, IL-17A, IL-17E/IL-25, IL-17F, IL-18, IL-22, IL-27, MCP-1, M-CSF, MIG, MIP-1 $\alpha$, PDGF-AA,
PDGF-AB/BB, RANTES, TGF $\alpha$, TNF $\beta$, and VEGF-A) showed no group differences (data not shown). Overall (no disease severity grouping; Fig. 2A-J), IL-6 levels were significantly higher in men vs. women in the COVID groups (Fig. 2B). When compared with controls, Eotaxin in men, and MCP-3 in women were significantly lower (Fig. 2C, E), and IL-10 in women was higher (Fig. 2D), a control vs. COVID-19 difference that 

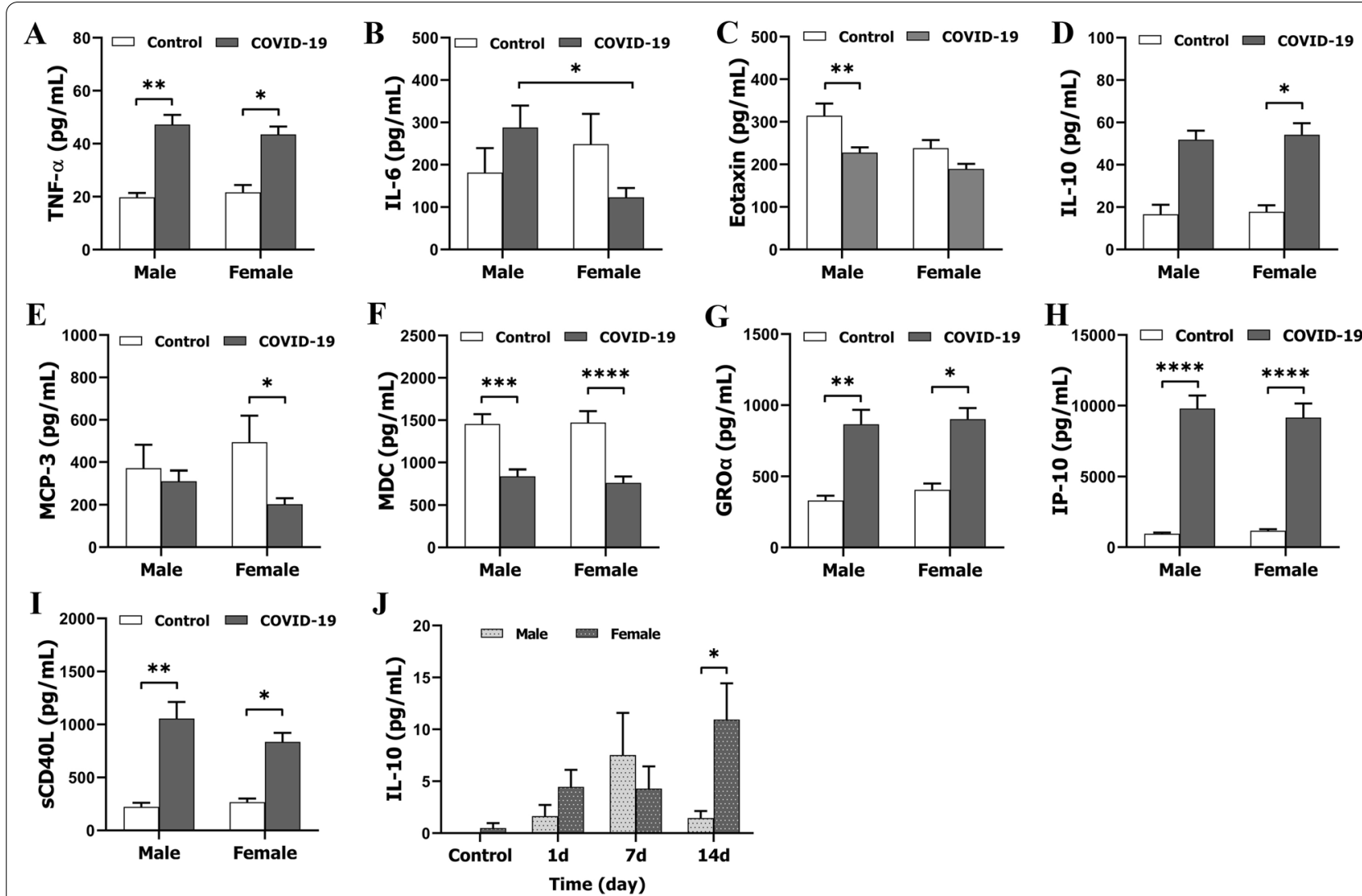

Fig. 2 Levels of inflammatory mediators in control vs. COVID-19 plasma samples. A-I Levels of TNF-a, IL-6, Eotaxin, IL-10, MCP-3, MDC (CCL22), GROa, IP-10, and SCD40L, respectively, in Cohort 1 patients measured by MultiPlex ELISA. J Levels of anti-inflammatory IL-10 cytokine in Cohort 2 patients by conventional ELISA. $N=6-88$ independent experiments assayed in duplicates. ${ }^{*} p<0.05 ;{ }^{* *} p<0.01{ }^{* * *} p<0.001$, ${ }^{* * *} p<0.0001$; two-way ANOVA with Sidak's post hoc correction

was not seen in the counterpart sex, respectively. No sex differences were found in levels of MDC (CCL22), GRO $\alpha$, IP-10, and sCD40L, although males and females exhibited synchronous changes compared to controls (Fig. 2F-I). For samples from Cohort 2 patients, three pro- (IL-6, IL-8, TNF- $\alpha$ ) and three anti-inflammatory mediators (IL-10, TGF- $\beta 1$, CD200) were assayed by conventional ELISA. Sex differences were only seen in IL-10 levels, which were significantly higher in females vs. males at 14 days (Fig. 2J). Together with the data from the cohort 1 patients (Fig. 2D), this suggests that a more robust anti-inflammatory response was seen in female vs. male COVID-19 patients.

\section{Sex differences in the levels of inflammatory markers are dependent on the severity of COVID-19 illness}

The symptoms of COVID-19 are variable, ranging from mild fever to severe illness and intensive care unit (ICU) admission. The severity of the disease may be responsible for the cytokine profiles. We examined if the sex differences seen in the above overall data (Fig. 2) remained in patients with different disease severity (mild, moderate and severe). Interestingly, we found that the most dramatic sex differences were seen in the severe groups, e.g., males in the severe group had significantly higher levels of IL-6, IL-8, and MCP-1 than females (Fig. 3B, C, $\mathrm{L}$ ), a sex difference not seen in mild or moderate group. Males with severe disease also had significantly higher levels of GRO $\alpha, \mathrm{sCD} 40 \mathrm{~L}$, and MIP-1 $\beta$ than control, mild, or moderate male groups, which was not seen in females (Fig. 3G, I, J). Of note, compared to controls, the antiinflammatory cytokine, IL-10, significantly increased in females with moderate and severe disease, but was not significantly elevated until the severe stage in males (Fig. 3E). For TNF- $\alpha$, Eotaxin, MDC (CCL22), IP-10, and Fractalkine, the data revealed no sex differences. Taken together, male COVID-19 patients with severe illness have an exacerbated immune response compared to females with severe disease. 

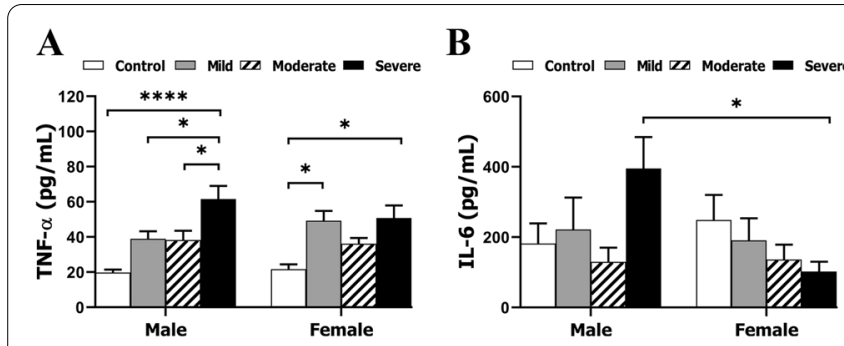

C

D

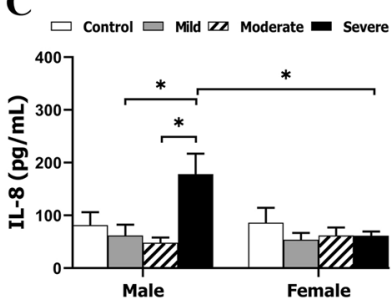

D $\square$ Control $\square$ Mild $\square$ Moderate $\square$ Severe
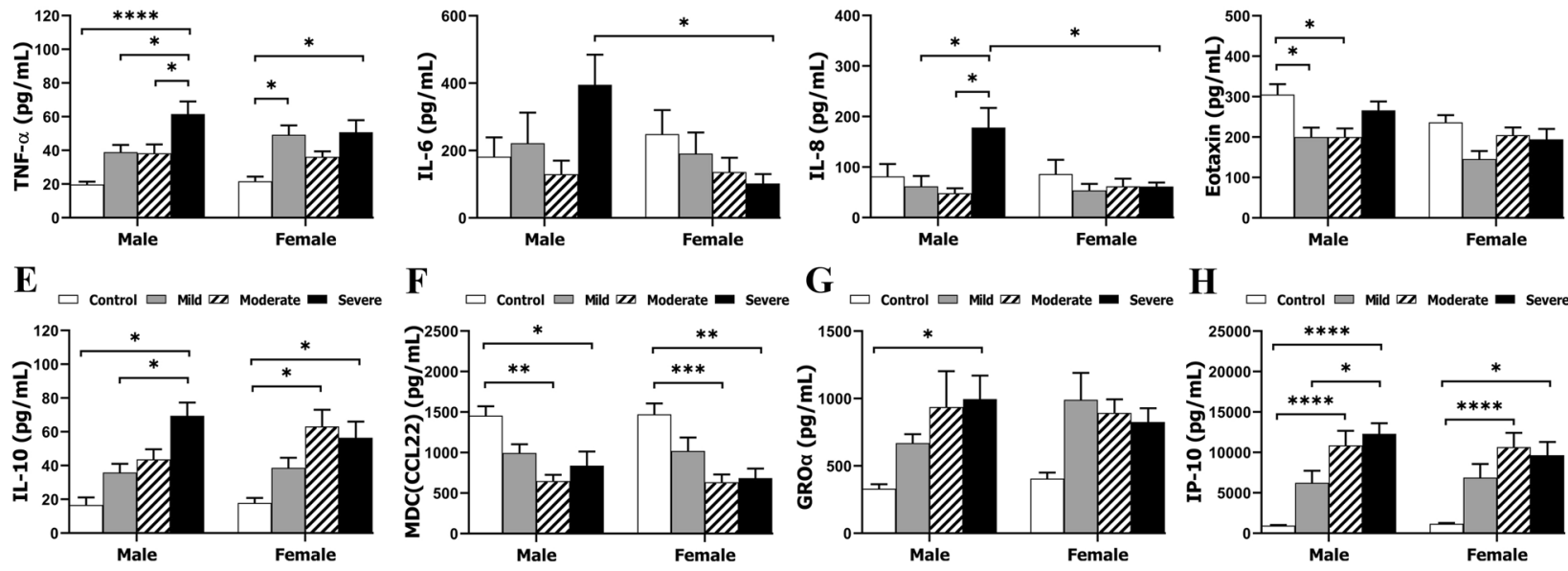

I

$\mathbf{J}$

K
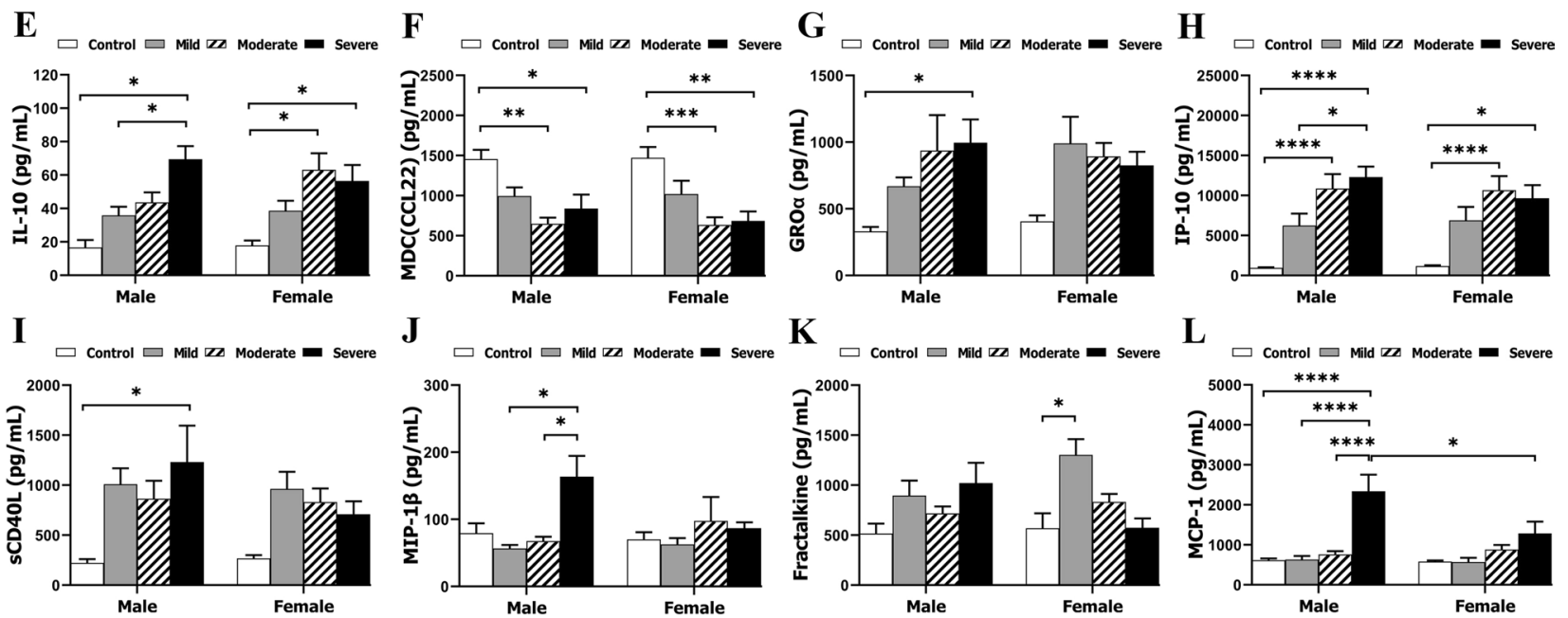

$\mathbf{L}$

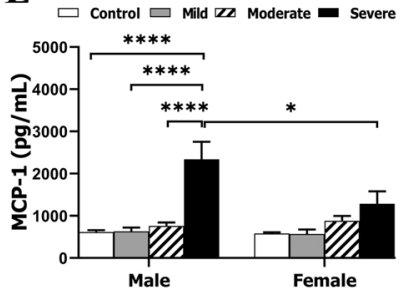

Fig. 3 Levels of inflammatory mediators in control vs. COVID-19 plasma samples based on disease severity. A-L levels of TNF-a, IL-6, IL-8,

Eotaxin, IL-10, MDC (CCL22), GROa, IP-10, sCD40L, MIP-1 $\beta$, Fractalkine, and MCP-1, respectively, in Cohort 1 patients by MultiPlex ELISA. N=6-33

independent experiments assayed in duplicates. ${ }^{*} p<0.05$; ${ }^{* *} p<0.01{ }^{* * *} p<0.001$, ${ }^{* * *} p<0.0001$; two-way ANOVA with Sidak's post hoc correction

\section{Sex differences in circulating immune cells}

To further evaluate the immune response to COVID19 , we performed flow cytometry on fresh blood samples to examine leukocytes from Cohort 2 patients at three time points ( $24 \mathrm{~h}, 7$ days, and 14 days) since hospital admission. A panel of antibodies to detect neutrophils (Table 1), monocytes (Table 2), and lymphocytic $\mathrm{T}$ and $\mathrm{B}$ cells (Table 3 ) was used to identify these leukocyte subsets. Active neutrophils were gated as CD11b ${ }^{+}$DEspR ${ }^{+}$[17-19] (Fig. 4A), monocytes as $\mathrm{CD} 11 \mathrm{~b}^{+} \mathrm{CD} 45^{+} \mathrm{CD} 14^{+} \mathrm{CD} 24^{-}$(Fig. $4 \mathrm{~B}$ ), $\mathrm{T}$ cells as $\mathrm{CD}_{4} 5^{+} \mathrm{CD}^{+}$and $\mathrm{B}$ cells as $\mathrm{CD} 45^{+} \mathrm{CD} 19^{+}$(Fig. 4C). Interestingly, sex differences were found in both myeloid cells (neutrophils/monocytes) and lymphocytes, but exhibited different patterns by sex. Male patients had more neutrophils and monocytes at each time point (all significant except a trend at $24 \mathrm{~h}$ for monocytes) than female patients (Fig. 4D, E). However, there was a significant elevation in B cells in females vs. males at both 7 days and 14 days (Fig. 4F). No sex difference was seen in $\mathrm{T}$ cells (Fig. 4G).

\section{Pneumonia and other organ involvement}

After infection with COVID-19, patients often have signs of other organ involvement or comorbidities in addition to pneumonia. We reviewed the hospitalization history of our patients and analyzed organ involvement. Figure $5 \mathrm{~A}$ shows the percentage of pneumonia, sepsis, acute kidney injury (AKI), deep vein thrombosis (DVT), and myocardial infarction in all patients. Overall, more male patients develop these complications than females, with significant differences in pneumonia and AKI. Figure 5B and C shows the percentages of these diseases in Cohort 1 and Cohort 2 patients, respectively. In males with moderate disease, the percentage of pneumonia was significantly higher than that of females. Prevalence of sepsis showed an increasing trend across the severity of COVID-19 infection in males $(p=0.012)$ but not in females.

\section{Discussion}

The immune response to viral infection plays an important role in tissue damage caused by COVID-19 [20]. In the present study, we analyzed the immune response in patients with acute COVID-19 respiratory tract infection 


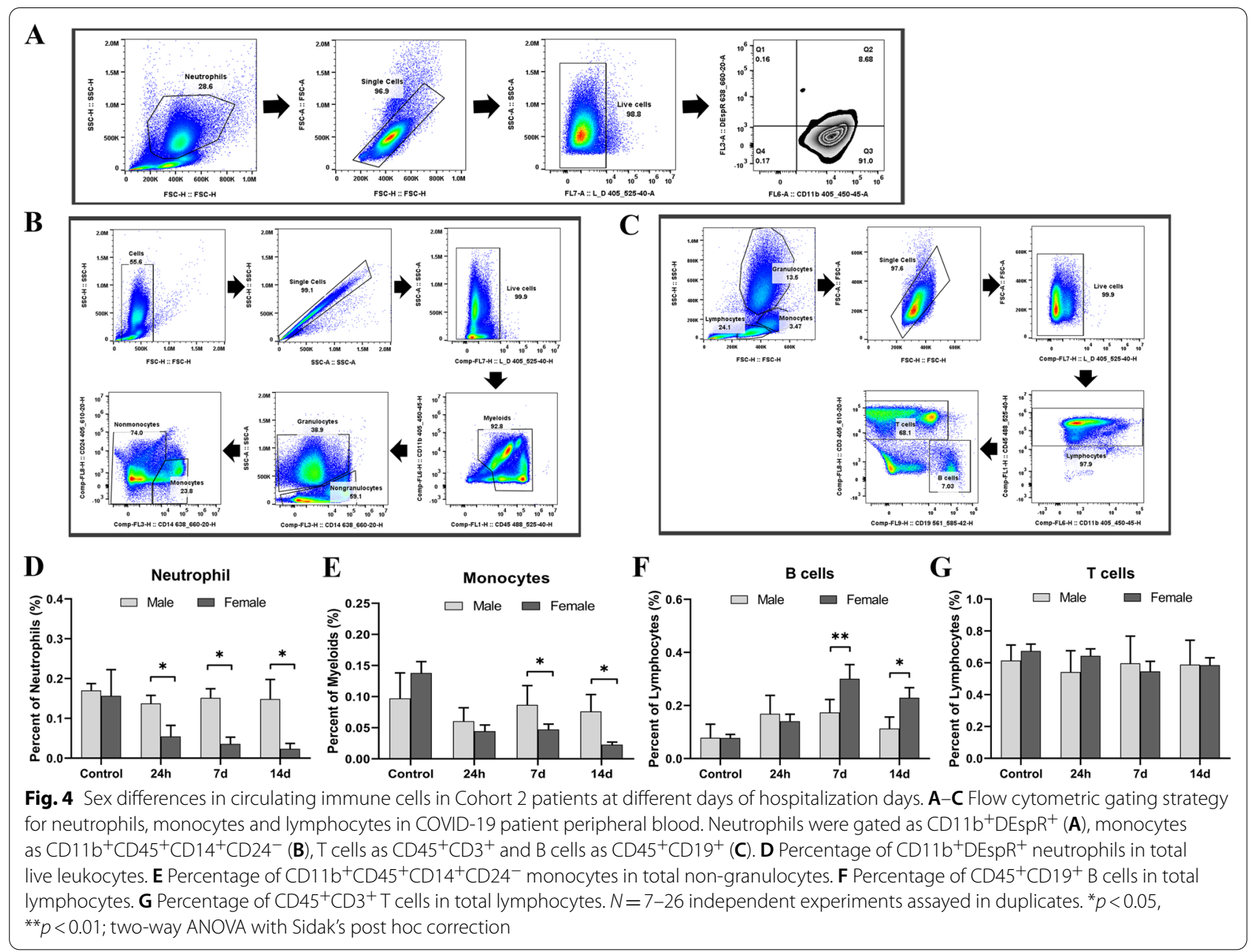

both at the cytokine level and the cellular level, and found several novel findings. Firstly, in patients hospitalized with COVID-19, as seen by others, men were overrepresented, and overall had more robust inflammation than females, a sex difference that was particularly pronounced in patients with severe disease. Secondly, although female patients also showed a pro-inflammatory response, they appear to mount an anti-inflammatory response earlier than male patients. Thirdly, our data suggest that early innate immune responses to COVID19 are more pronounced in men, and delayed adaptive immunity is more highly activated in women. To increase the accuracy and precision in our data interpretation, we analyzed the immune response longitudinally and also disaggregated the data based on the severity of the disease; therefore, we believe our data are convincing and reflect the nature of the immune response in acute COVID-19 respiratory tract infection patients.

Previous clinical data have shown that although the prevalence of infection is the same in men and women, men are more likely to be hospitalized, have a more severe disease course, and higher mortality than women [21]. Our findings are consistent with these reports, as male patients outnumbered females not only in total numbers (350 vs. 276), but also in most age and ethnic groups, and males had higher mortality than females (Fig. 1). In addition, more male patients developed pneumonia, sepsis, AKI, DVT and myocardial infarction than females after COVID-19 infection. A number of reasons have been postulated for the sex differences in the response to COVID-19. Some studies have shown that men have a higher level of ACE2 receptors, used for viral entry into target cells. The cellular serine protease TMPRSS2, responsible for coronavirus spike (S) protein priming, is also highly expressed in the prostate epithelium and is sensitive to androgens [22]. Differences in hormone levels may also underlie the observed sex differences [11]. The present study provides evidence that in addition to the above, the differential immune responses seen in male vs. female patients may also play an important role in mediating the sex differences seen in COVID19 patients. 


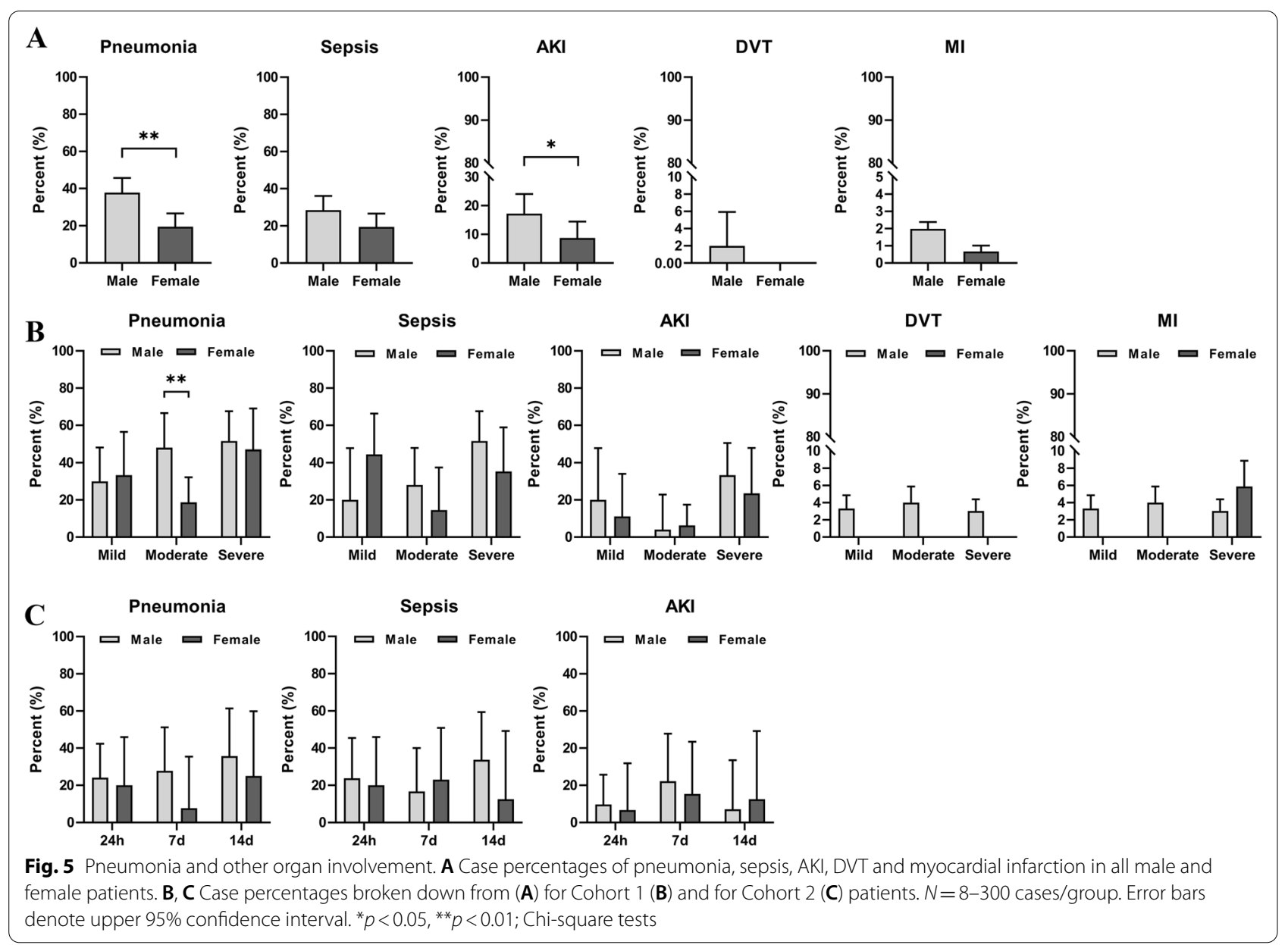

Previous studies have found that more severe clinical symptoms of COVID-19 were associated with elevated levels of inflammatory markers [23] and predict poorer prognosis and mortality [24]. In the present study, circulating levels of inflammatory mediators appear to reflect the systemic inflammation caused by the infection. It is intriguing that sex differences in the cytokine levels were found both in the overall data (Fig. 2) and in the graded groups based on disease severity (Fig. 3). Although the overall data provided limited information as the cytokine levels were calculated regardless of the disease severity, sex differences were evident in the pro-inflammatory cytokine IL-6 (higher in males) and the anti-inflammatory cytokine IL-10 (higher in females). When we analyzed the data in the graded groups (Fig. 3), more sex differences were revealed, especially in the severe group. Of the 12 inflammatory mediators, 6 (IL-6, IL-8, GRO $\alpha$, sCD4L, MIP-1 $\beta$, MCP-1) exhibited very high levels in males with severe disease but not in females, all of which are pro-inflammatory mediators. The anti-inflammatory cytokine IL-10 again exhibited a female favorable phenotype, i.e., the level significantly increased in the moderate group but not in the males compared to controls. IL-10 is well known for its beneficial roles in inflammation resolution and tissue repair in inflammatory diseases [25]. The higher level of IL-10 in female vs. male patients may be an important reason why female patients have a better prognosis and lower mortality.

Our data also revealed a sex difference in the leukocyte response to acute COVID-19 respiratory tract infection. While the innate immune response seemed to be more active in male patients indicated by higher numbers of circulating inflammatory neutrophils and monocytes, the adaptive immune response was more robust in females, and females had significantly more B cells at 7 days and 14 days (Fig. 4F). B cells possess distinct machinery for adaptive immunity compared to other immune cells. Upon activation, B cells proliferate rapidly and differentiate into antibody-secreting plasma cells; antibodies released from these cells bind specifically to foreign antigens (e.g., viruses) and inactivate viruses and microbial toxins [26]. In addition to antibody secretion, B cells also regulate $\mathrm{T}$ cell responses affecting the progression of diseases; therefore, B cell-induced adaptive immunity is 
both cellular and humoral [27]. On the other hand, neutrophils are a major component of the innate immune response [28] and monocytes/macrophages are members of the mononuclear phagocytic system, are also a component of innate immunity [29]. While the innate immune response is immediate, the effect of the adaptive immune response is long-lasting and highly specific. Another cell type that participates in adaptive immunity is the $\mathrm{T}$ cell. Somewhat surprisingly, we did not see any significant sex difference in T cells in our data (Fig. 4G). Although both innate and adaptive immunity are important in the fight against foreign antigens, the present study suggests that the adaptive immunity in female patients is more robust and sustained in females compared to male patients. The chronic consequences of this sex-specific activation of $B$ cells remain unknown.

The present study has some limitations that should be kept in mind when interpreting the data. The age of all recruited patients ranges from 19 to 101, and aged patients may have more complications; however, our control participants were healthy with an age range of 25-60. Therefore, age-matching between controls vs. patients was not ideal. We also did not measure circulating sex hormone levels (estrogen and testosterone) in our patients; therefore, the contribution of the gonadal hormones to the sex difference seen in immunity is not known. We also did not deeply phenotype the immune cells, and contributions of specific lower prevalence cells will require further study. Of note, the difference in immune responses between males vs. females may not be solely related to acute lung injury (pneumonia); instead, it may reflect a systemic response, as males also had higher incidence of other organ involvement than females (Fig. 5).

\section{Perspectives and significance}

In conclusion, the present study examined sex differences in patients with acute COVID-19 respiratory tract infection, with a focus on the immune responses. Examining inflammatory mediators, we found female patients launched a more robust anti-inflammatory response than males. Using flow cytometry to analyze immune cells, the study revealed that innate and adaptive immunity were differentially activated in male vs. female patients. Although these sex differences in the immune response could either be resultant or causative to the COVID-19 virus infection, we conclude that the "female favorable" phenotype of COVID-19 could be at least in part due to the higher levels of anti-inflammatory factors and earlier activation of adaptive immunity.

\section{Abbreviations}

COVID-19: Coronavirus Disease 2019; SARS-CoV-2: Severe acute respiratory syndrome coronavirus 2; ICU: Intensive Care Unit; DEspR: Dual endothelin-1/ VEGF signal peptide receptor; ACE2: Angiotensin converting enzyme 2; TLR7 Toll-like receptor 7; MCP-3: Monocyte-chemotactic protein-3; MDC (CCL22): Macrophage-derived chemokine (CCL22); GROa: Growth-related oncogene a; IP-10: Interferon gamma-induced protein 10; sCD40L: Soluble form of CD40L; MIP-1 $\beta$ : Macrophage inflammatory protein-1 $\beta$; MCP-1: Monocyte chemoattractant protein-1.

\section{Supplementary Information}

The online version contains supplementary material available at https://doi. org/10.1186/s13293-021-00410-2.

Additional file 1. Inclusion and exclusion criteria.

Additional file 2. Inflammatory Mediator list.

\section{Acknowledgements}

We thank all study participants for the generous donation of blood samples, and acknowledge members of the BRAINS Research Laboratory for thoughtful discussion and suggestions on the data analysis and result interpretation.

\section{Authors' contributions}

SQ, and CN: project conception and design, conducting experiments, acquisition of data, analysis and interpretation of data, and manuscript writing. MSDA: project conception, conducting experiments and acquisition of data. AAM: conducting experiments and acquisition of data. FCE: conducting experiments. AHW, RS, DCG, and HAC: collecting and processing blood samples. YK: analysis of data and writing. LDM, and FL: project conception and design, interpretation of data and manuscript writing. All authors read and approved the final manuscript.

\section{Funding}

This work was supported by NIH Grants: R01 NS108779 and R01 MH127856 (F.L./L.D.M.) and the Huffington Foundation (L.D.M.).

\section{Declarations}

Ethics approval and consent to participate

Ethics approval for use of human blood samples in this study was obtained from the Memorial Hermann Texas Medical Center with appropriate ethics committee approval (Committee for Oversight of Research and Clinical Training Involving Decedents). Informed written consent was obtained from all study participants. The study was approved by the Institutional Review Board (IRB) of University of Texas Health Science Center at Houston.

Consent for publication

Not applicable.

\section{Competing interests}

The authors declare that they have no competing interests.

\section{Author details}

${ }^{1}$ Department of Neurology, McGovern Medical School, The University of Texas Health Science Center at Houston, 6431 Fannin Street, Houston, TX 77030, USA. ${ }^{2}$ Department of Neurosurgery, McGovern Medical School, The University of Texas Health Science Center at Houston, 6431 Fannin Street, Houston, TX 77030, USA.

Received: 1 July 2021 Accepted: 13 December 2021

Published online: 20 December 2021

\section{References}

1. Johns-Hopkins-University. The Johns Hopkins Coronavirus Resource Center. [5/27/2021]. Available from: https://coronavirus.jhu.edu/. 
2. Chowdhury MA, et al. Immune response in COVID-19: a review. J Infect Public Health. 2020;13(11):1619-29.

3. Fara A, et al. Cytokine storm and COVID-19: a chronicle of pro-inflammatory cytokines. Open Biol. 2020;10(9):200160.

4. Abate BB, et al. Sex difference in coronavirus disease (COVID-19): a systematic review and meta-analysis. BMJ Open. 2020;10(10):e040129.

5. Meng Y, et al. Sex-specific clinical characteristics and prognosis of coronavirus disease-19 infection in Wuhan, China: a retrospective study of 168 severe patients. PLoS Pathog. 2020:16(4):e1008520.

6. Leung GM, et al. The epidemiology of severe acute respiratory syndrome in the 2003 Hong Kong epidemic: an analysis of all 1755 patients. Ann Intern Med. 2004;141(9):662-73.

7. Scully EP, et al. Considering how biological sex impacts immune responses and COVID-19 outcomes. Nat Rev Immunol. 2020;20(7):442-7.

8. National Institutes of Health. COVID-19 treatment guidelines panel. Coronavirus Disease 2019 (COVID-19) treatment guidelines. Clinical spectrum of SARS-CoV-2 infection. [6/29/2021]. Available from: https://www.covid 19treatmentguidelines.nih.gov/overview/clinical-spectrum/.

9. National Institutes of Health. COVID-19 treatment guidelines panel. Coronavirus Disease 2019 (COVID-19) treatment guidelines. Therapeutic management of adults with COVID-19. [6/29/2021]. Available from: https://www.covid19treatmentguidelines.nih.gov/management/thera peutic-management/.

10. Takahashi T, et al. Sex differences in immune responses that underlie COVID-19 disease outcomes. Nature. 2020;588(7837):315-20.

11. Gebhard C, et al. Impact of sex and gender on COVID-19 outcomes in Europe. Biol Sex Differ. 2020;11(1):29.

12. Gianicolo EAL, et al. Gender specific excess mortality in Italy during the COVID-19 pandemic accounting for age. Eur J Epidemiol. 2021. https:// doi.org/10.1007/s10654-021-00717-9.

13. Gomez JMD, et al. Sex differences in coronavirus disease 2019 (COVID-19) hospitalization and mortality. J Womens Health (Larchmt). 2021. https:// doi.org/10.1089/jwh.2020.8948.

14. Nielsen J, et al. Sex-differences in COVID-19 associated excess mortality is not exceptional for the COVID-19 pandemic. Sci Rep. 2021;11(1):20815.

15. Jose RJ, Manuel A. COVID-19 cytokine storm: the interplay between inflammation and coagulation. Lancet Respir Med. 2020;8(6):e46-7.

16. Hojyo S, et al. How COVID-19 induces cytokine storm with high mortality. Inflamm Regen. 2020;40:37.

17. Ruiz-Opazo N, et al. Molecular characterization of a dual endothelin-1/ angiotensin II receptor. Mol Med. 1998;4(2):96-108.

18. Herrera $V L$, et al. Embryonic lethality in Dear gene-deficient mice: new player in angiogenesis. Physiol Genomics. 2005;23(3):257-68.

19. Herrera $\mathrm{VL}$, et al. Sex-specific hippocampus-dependent cognitive deficits and increased neuronal autophagy in DEspR haploinsufficiency in mice. Physiol Genomics. 2008;35(3):316-29.

20. Kasuga $Y$, et al. Innate immune sensing of coronavirus and viral evasion strategies. Exp Mol Med. 2021;53(5):723-36.

21. Alkhouli M, et al. Sex differences in case fatality rate of COVID-19: insights from a multinational registry. Mayo Clin Proc. 2020;95(8):1613-20.

22. Hoffmann M, et al. SARS-CoV-2 cell entry depends on ACE2 and TMPRSS2 and is blocked by a clinically proven protease inhibitor. Cell. 2020;181(2):271-280.e8.

23. Huang C, et al. Clinical features of patients infected with 2019 novel coronavirus in Wuhan, China. Lancet. 2020;395(10223):497-506.

24. Liu QQ, et al. Cytokines and their relationship with the severity and prognosis of coronavirus disease 2019 (COVID-19): a retrospective cohort study. BMJ Open. 2020;10(11):e041471.

25. Steen $\mathrm{EH}$, et al. The role of the anti-inflammatory cytokine interleukin-10 in tissue fibrosis. Adv Wound Care (New Rochelle). 2020;9(4):184-98.

26. Sette A, Crotty S. Adaptive immunity to SARS-CoV-2 and COVID-19. Cell. 2021;184(4):861-80

27. Sage AP, et al. The role of $B$ cells in atherosclerosis. Nat Rev Cardiol. 2019;16(3):180-96.

28. Selders GS, et al. An overview of the role of neutrophils in innate immunity, inflammation and host-biomaterial integration. Regen Biomater. 2017:4(1):55-68

29. Chiu S, Bharat A. Role of monocytes and macrophages in regulating immune response following lung transplantation. Curr Opin Organ Transplant. 2016;21(3):239-45.

\section{Publisher's Note}

Springer Nature remains neutral with regard to jurisdictional claims in published maps and institutional affiliations.
Ready to submit your research? Choose BMC and benefit from:

- fast, convenient online submission

- thorough peer review by experienced researchers in your field

- rapid publication on acceptance

- support for research data, including large and complex data types

- gold Open Access which fosters wider collaboration and increased citations

- maximum visibility for your research: over $100 \mathrm{M}$ website views per year

At BMC, research is always in progress.

Learn more biomedcentral.com/submissions 